\title{
Small bowel perforation secondary to foreign body ingestion mimicking appendicitis: a case report
}

\author{
Bijit B. Medhi*, P. D. Gupta, Fahad Tauheed, Vikram Singh Chauhan
}

Department of General Surgery, SMS\&R, Sharda University, Greater Noida, UP, India

Received: 28 May 2021

Revised: 01 August 2021

Accepted: 03 August 2021

*Correspondence:

Dr. Bijit B Medhi,

E-mail: zeet.b.medhi@gmail.com

Copyright: (c) the author(s), publisher and licensee Medip Academy. This is an open-access article distributed under the terms of the Creative Commons Attribution Non-Commercial License, which permits unrestricted non-commercial use, distribution, and reproduction in any medium, provided the original work is properly cited.

\begin{abstract}
Admittance in the emergency room with complaints of acute abdominal pain is a common scenario. Patients who see the doctor due to foreign object ingestion present transitory symptomatology. In most cases, foreign object ingestion in adults is accidental, although it may be linked to physiological, anatomical, mechanical, social and psychiatric factors. The frequency of voluntarily ingested objects is higher among children and teenagers than in any other age group. Few people go to the doctor because of persistent clinical manifestations or secondary manifestations of previous complications. In most cases, the foreign body is found incidental to another medical procedure. Here we report a case of small bowel perforation due to ingestion of a sharp foreign body in a 2 years old child. We further elaborate that how, in paediatric age group these conditions are frequently misdiagnosed and usually present late to us. Hence, the increasing need and importance of a thorough pre-op workup, especially in the pediatric age group.
\end{abstract}

Keywords: Foreign body ingestion, Small bowel, Perforation, Mimicking, Appendicitis

\section{INTRODUCTION}

Accidental ingestion of foreign bodies is a common condition in clinical practice. However, small bowel perforation due to ingestion of foreign bodies is uncommon. Foreign body (FB) ingestion is very common in children and most events occur in children between 6 months and 3 years of age. Notably, $80 \%-90 \%$ FBs in the gastrointestinal (GI) tract are passed spontaneously without any complications, $10 \%-20 \%$ are removed endoscopically and $1 \%$ require surgery secondary to complications. ${ }^{1}$ The ingestion of foreign body is rarely associated with intestinal perforation, even if a sharp object is swallowed. ${ }^{2}$ The alimentary canal is remarkably resistant to perforation. About $20 \%$ of the foreign bodies fail to pass through the entire GI tract. Any FB that remains in the tract may cause obstruction, perforation or haemorrhage and fistula formation. Less than $1 \%$ result in perforations from the mouth to the anus and those are mostly caused by sharp objects and erosions. ${ }^{3}$ Of these sharp objects chicken bones and fish bones account for half of the reported perforations. The most common sites of perforations are the ileo-caecal junction and the sigmoid colon. $^{2}$

\section{CASE REPORT}

A 2 years old child presented to the casualty with complaints of loose stools and abdominal distension for 3 days. After initial examination and investigations, a diagnosis of appendiceal or ileal perforation was made. The patient was taken up for exploratory laparotomy for intestinal perforation after resuscitation. A sharp foreign body was found perforating the proximal jejunum at its mesenteric border. Primary repair was done after removal of the foreign body. 


\section{Presentation of the case}

A 2 years old male child presented to the casualty with complaints of loose stools and abdominal distension for 3 days. The history obtained by child's mother suggests that the child starts to cry after his meals and peculiarly rubbed his abdomen while crying.

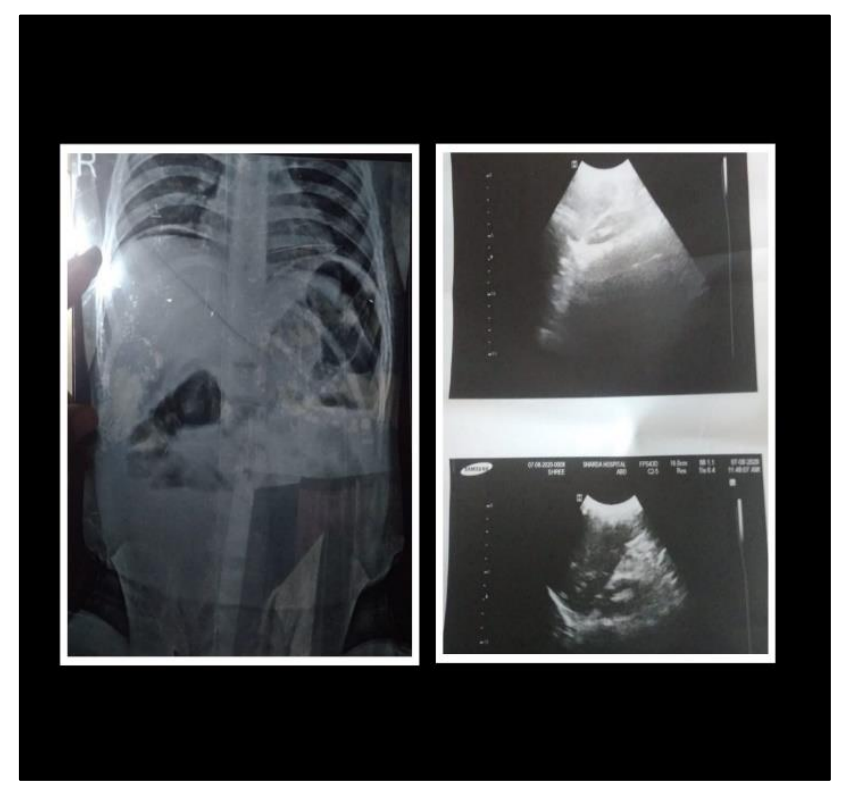

Figure 1: X-ray abdomen (erect) showing free gas under the diaphragm and free fluid interspersed in between bowels in the USG abdomen.

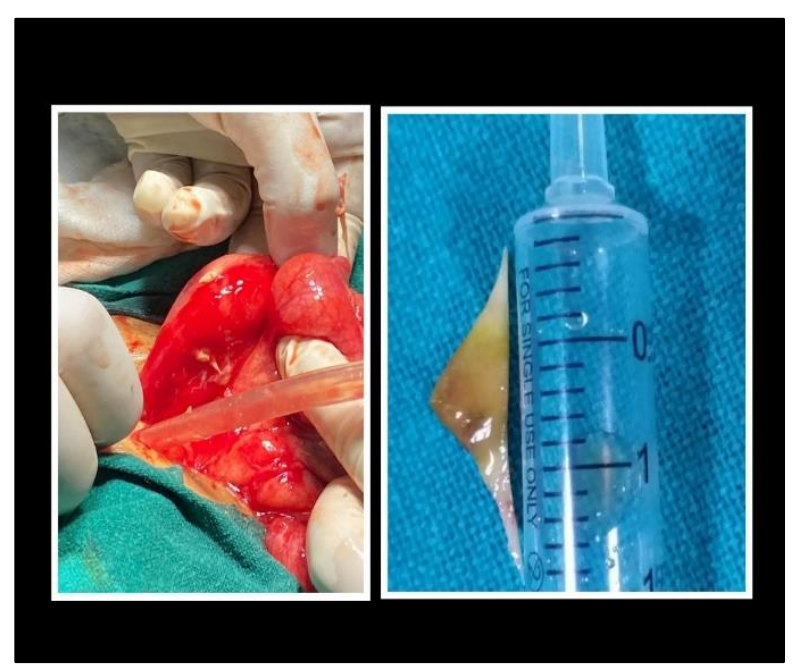

Figure 2: Sharp foreign body can be viewed protruding out of the jejunum and the bone piece for size.

On examination the abdomen of the child was distended but there was no guarding or rigidity and the bowel sounds were heard on auscultation. The child was not febrile. An Ultrasonogram of the abdomen revealed free fluid in the abdomen interspersed between the bowels and suggested of intestinal obstruction. An x-ray abdomen, however, revealed free air under the diaphragm suggesting hollow viscous perforation. The patient was taken up for exploratory laparotomy, based on the $\mathrm{x}$-ray findings and clinical symptoms although clinically he didn't show any signs of peritonitis. His pre-op hemogram showed a raised TLC of $13,000 / \mathrm{cmm}$.

\section{Per-operative}

The patient was taken up for emergency laparotomy. The abdomen was accessed through a infra-umbilical transverse incision extending to the right side based on the child's history of rubbing that part of the abdomen after meals. The peritoneal cavity was entered and pus flakes were noticed upon opening it with moderate feco-purulent free fluid in the peritoneum.

Inspection of the small bowel led to the discovery of a hard, solid, sharp and pointed object in the jejunum, approximately $30 \mathrm{~cm}$ from the duodeno-jejunal flexure, with a puncture perforation of the jejunum from inside the bowel in its mesenteric border. Upon examination the object was felt approximately to be $3 \times 1 \mathrm{~cm}$. The perforation appeared to be occupied by the object itself causing perforation to be obscured. (Figure 2)

The foreign body was removed through the perforation site in the jejunum. It appeared to be a T shaped bone piece. The edges of the small bowel were freshened and primary repair done with 3-0 PDS. A 18fr pelvic drain was put in the pelvic cavity and anchored with 3.0 ethilon. After a thorough peritoneal lavage with warm Saline was done and the abdomen was closed in layers.

\section{Post-operative}

The post-operative recovery of the patient was uneventful. The child was allowed orally on the 2 nd post-operative day after he passed stools. He tolerated oral feeds well without any pain or distention. There was no sign of abdominal discomfort post-operatively and drain was removed on day 3. He was discharged on day 4 on oral antibiotics and analgesics.

\section{DISCUSSION}

Intestinal perforation secondary to a foreign body presents as an acute abdominal emergency, which may mimic acute appendicitis or diverticulitis. ${ }^{6}$ Patients often develop abdominal pain, nausea, vomiting, and fever after perforation. Some patients are even operated on for acute appendicitis, especially, those with terminal ileal perforation. The foreign body is usually found during the preoperative work-up or during surgery. ${ }^{7,8}$

Our patient being of paediatric age group couldn't exactly locate his pain but as the child's mother stated he used to rub his right lower quadrant and cry after meals we could assume he presented with right lower quadrant pain and significantly increased white blood cell counts. 
Interestingly, imaging tests showed free air under the diaphragm leading us to believe it may be an intestinal perforation. We therefore first considered a diagnosis of appendiceal or ileal perforation.

Foreign body-associated intestinal perforation mimicking acute appendicitis has been reported before, and such perforations are typically repaired successfully. ${ }^{9}$ In our patient, the first symptom was abdominal pain, which was probably caused directly by the foreign bodies. The foreign body could not be digested in the upper digestive tract and failed to be discharged and was therefore retained in the intestine. Hence time delay in the presentation of the symptoms. Foreign bodies usually pass through the esophagus without any complications. Owing to the thick wall and large volume of the stomach, foreign bodies generally do not cause gastric perforation. ${ }^{10}$ If patients who have swallowed a foreign body visit a hospital at this stage, the foreign bodies can be endoscopically removed, thus avoiding complications such as intestinal perforation. The risk of perforation is higher in the small intestine because of its relatively small lumen, especially if the foreign body is sharp and hard (example- chicken or fish bones). Although previous literature has suggested that the usual site for sharp foreign body perforation is the ileo-caecal junction and sigmoid colon. However there is no literature reporting on cases of sharp foreign body perforation of the jejunum in children. In our patient it was found that sharp foreign body was lodged in the proximal jejunum and initially may have cause the partial obstruction via the flapvalve mechanism but proceeded to perforation due to pressure on the sharp edge at the mesenteric border.

A reason for the initial misdiagnosis could be that a detailed history was not sought, and thus, the history of swallowing a foreign body before the onset of abdominal pain was missed in many cases. ${ }^{10}$ After recovery of the foreign body, the family recalled a history of cooking meat on the day before the onset of symptoms although they do not recall the child ingesting the same food.

\section{CONCLUSION}

The present case illustrates that in patients presenting to the ER with an acute abdomen it is not necessary for every case to be treated as acute appendicitis. A cumulative work up which includes detailed history, examination and radiography may help us reach our diagnosis and a precise and urgent plan of management can be decided, operative, if required. It is important to carefully ask for a history of foreign body ingestion (specifically a large or sharp foreign body). As more often than not a foreign body is not visualised on plain X-ray films, the aspect of obtaining a history of swallowing or accidental ingestion of foreign bodies almost becomes the cornerstone for further plan of management in such cases. Operating a patient for acute appendicitis and incidentally finding a perforation will not only lead to lengthened per op, which can include extension of the incision to increased blood loss and extended anaesthetic exposure, for the patient but also will complicate his post-operative recovery depending on the age and other co morbidities.

Funding: No funding sources Conflict of interest: None declared Ethical approval: Not required

\section{REFERENCES}

1. Seo JK. Endoscopic management of gastrointestinal foreign bodies in children. Indian $\mathrm{J}$ Pediatr. 1999;66(1 Suppl):S75-80.

2. Cleator IG, Christie J. An unusual case of swallowed dental plate and perforation of the sigmoid colon. $\mathrm{Br}$ J Surg. 1973;60(2):163-5.

3. Perelman $H$. Tooth pick perforations of the gastrointestinal tract. J Abdom Surg. 1965;51-3.

4. Ashraf O. Foreign body in the esophagus: a review. Sao Paulo Med J. 2006;124(6):346-9.

5. Incidental foreign body in the gastrointestinal tract. Report of three cases and literature review. Rev Col Gastroenterol. 2011;26:4.

6. Akhtar S, McElvanna N, Gardiner K, Irwin S. Bowel perforation caused by swallowed chicken bones: A case series. Ulster Med J. 2007;76(1):37-8.

7. Madrona AP, Hernández JAF, Prats MC. Intestinal perforation by foreign bodies. Eur J Surg. 2000;166:307-9.

8. Hsu SD, Chan DC, Liu YC. Small-bowel perforation caused by fish bone. World J Gastroenterol. 2005;11:1884-5.

9. Perko Z, Bilan K, Pogorelić Z. Acute appendicitis and ileal perforation with a toothpick treated by laparoscopy. Coll Antropol. 2008;32:307-9.

10. Almoudaris. Fish bone perforation mimicking acute appendicitis. J Med Cases. 2011;2:296-9.

11. Small bowel perforation secondary to foreign body ingestion mimicking acute appendicitis. Medicine (Baltimore). 2019;98(30):e16489.

Cite this article as: Medhi BB, Gupta PD, Tauheed F, Chauhan VS. Small bowel perforation secondary to foreign body ingestion mimicking appendicitis: a case report. Int Surg J 2021;8:2807-9. 